\title{
Genetic dimension of the coevolution of virulence-resistance in Drosophila - parasitoid wasp relationships
}

\author{
S Dupas ${ }^{1}$, Y Carton ${ }^{1}$ and M Poiriè ${ }^{2}$ \\ ${ }^{1}$ Laboratoire Populations, Génétique et Evolution, CNRS 91198, Gif-sur-Yvette Cedex, France; ${ }^{2} I R B I$, Université François Rabelais, \\ 37200 Tours, France
}

\begin{abstract}
Variations observed in parasite virulence and host resistance may be the outcome of coevolutionary processes. Recent theoretical developments have led to a 'geographic mosaic theory' of coevolution according to which there are some localities where reciprocal selection occurs (hot spots) and others where it is strongly reduced (cold spots). Studies of host-parasitoid systems back this up, revealing a geographical variation of traits subjected to antagonistic selection governed by variations in the strength of the ecological interactions. A more detailed analysis of the genetic basis of these geographic variations in a model system - the interaction between Drosophila melanogaster and its specific parasitoid Leptopilina boulardi - suggests that cold spots and
\end{abstract}

hot spots are also driven by the amount of genetic variation available for the trait considered. Our approach, based on isolating reference strains, has been found to predict the result of sympatric interactions and it will be helpful in identifying the selective forces responsible for the coevolution. In this model, host resistance to a standardised reference strain is a weak predictor of the outcome of interactions in the field, and the main parameter accounting for the geographic variations is the number of host species available, with less parasitoid virulence towards $D$. melanogaster being found in areas displaying a more diversified host community.

Heredity (2003) 90, 84-89. doi:10.1038/sj.hdy.6800182

Keywords: parasitoids; Drosophila; resistance; virulence; coevolution; geographic mosaics; genetic interactions

\section{Introduction}

The impact of parasitoids on host mortality (Godfray, 1993) results in strong selective pressures and antagonistic interactions between the mechanisms responsible for host resistance and for parasitoid virulence, respectively. Insects infested with parasitoids initiate a haemocyte-mediated response (a melanotic capsule) that quickly destroys the intruders (Carton and Nappi, 1997, 2001), but parasitoids have developed various passive or active mechanisms to counteract this immune response (Strand and Pech, 1995; Godfray 2000). The possibility that these systems may coevolve is a recurrent question in evolutionary ecology (Kraaijeveld et al, 1998; Kraaijeveld and Godfray, 1999). Thompson (1999) has proposed that the selective interactions between species commonly differ between populations, resulting in coevolutionary 'hot spots' and 'cold spots'. A 'hot spot' corresponds to a subset of communities that exhibit intense reciprocal selection. Conversely, a 'cold spot' is a set of communities in which reciprocal selection does not occur, because of the weakness of the ecological interactions between the species.

There is still little data on the pattern of geographic variation in host resistance and parasitoid virulence in sympatric populations, but the studies that are available

Correspondence: Y Carton, Laboratoire Populations, Génétique et Evolution, CNRS, 91198, Gif-sur-Yvette Cedex, France.

E-mail: carton@pge.cnrs-gif.fr

Received 7 January 2002; accepted 3 August 2002 suggest that host resistance and parasitoid virulence, both of which can vary over short distances, are geographically structured (Kraaijeveld and Van Alphen, 1994, 1995). We have used well-characterised reference strains of host and parasitoid to analyse the geographic distribution of resistance and virulence phenotypes, thus making it possible to integrate a genetic dimension for reciprocal selection into the mosaic theory of hot spots and cold spots'.

Wild populations of Drosophila melanogaster are attacked by various species of Hymenoptera, including parasitoids of the genus Leptopilina (Figitidae, Cynipoidea), with three species described in Europe and at least five species in Africa (unpublished data). Leptopilina boulardi was chosen as a model since it acts as a highly specialised parasitoid of the melanogaster subgroup, both in Europe and in Africa (Carton and Nappi, 1991). The comparison of geographic patterns of resistance and virulence in the D. melanogaster - L. boulardi system, including a large-scale survey of the encapsulation level in sympatric populations, raises the question of the definition of coevolutionary 'hot spots' and 'cold spots' in this host-parasitoid system, and makes it possible to suggest some hypotheses about their ecological or genetic origin.

\section{Materials and methods}

Source of natural populations and reference strains A list of the collection sites is given in Table 1. Sympatric populations of $D$. melanogaster and $L$. boulardi were 
Table 1 Collection sites and encapsulation rates

\begin{tabular}{|c|c|c|c|}
\hline Locality (country) & Sympatry & Resistance & Virulence \\
\hline Nasrallah (Tunisia) & $0 \%(355)$ & $49.2 \%(185)$ & $99.8 \%(127)$ \\
\hline Ribeiri prato (Brazil) & $2.5 \%(157)$ & $63.7 \%(235)$ & $100.0 \%(211)$ \\
\hline Lamto (Ivory Coast) & $13.6 \%(58)$ & $9.6 \%(104)$ & $49.8 \%(279)$ \\
\hline Brazzaville (Congo) & $57.9 \%(644)$ & $42.4 \%(201)$ & $25.8 \%(48)$ \\
\hline Petit Bourg (Guadeloupe) & $1 \%(591)$ & $68.8 \%(117)$ & $99.8 \%(616)$ \\
\hline Açores (Portugal) & $0 \%(28)$ & $75 \%(24)$ & $98.9 \%(51)$ \\
\hline Malaucène (France) & $2 \%(243)$ & $77.7 \%(269)$ & $100.0 \%(28)$ \\
\hline Hampton (USA) & $2.9 \%(68)$ & & $80.7 \%(62)$ \\
\hline Bathurst (Gambia) & $9.7 \%(35)$ & & $90.3 \%(39)$ \\
\hline Palau (Italy) & $0 \%(314)$ & & $100.0 \%(28)$ \\
\hline Torres Nova (Portugal) & $0 \%(56)$ & & $100.0 \%(30)$ \\
\hline Cousin (Les Seychelles) & $5 \%(89)$ & & $94.8 \%(49)$ \\
\hline Ein Gedi (Israel) & $0 \%(65)$ & & $100.0 \%$ \\
\hline Rohtak (India) ${ }^{\mathrm{a}}$ & & $39.3 \%(56)$ & \\
\hline Medvastö (Finland) ${ }^{\mathrm{a}}$ & & $9.4 \%(85)$ & \\
\hline Niamey (Niger) ${ }^{a}$ & & $75.6 \%(82)$ & \\
\hline Seattle (USA) ${ }^{\mathrm{a}}$ & & $49.1 \%(61)$ & \\
\hline
\end{tabular}

The collection sites of D. melanogaster and L. boulardi populations are shown, as well as the rate of encapsulation (\%) in sympatric conditions, the level of resistance (\%) in D. melanogaster populations and the level of virulence $(\%)$ in L. boulardi populations (see Materials and methods for more details). ${ }^{a}$ Absence of L. boulardi. (no of larvae tested).

collected from different geographical locations throughout the parasitoid distribution area. L. boulardi was not found in four localities (see Table 1).

The origin of the host reference strain (Gif strain no. 940), which is homozygous for chromosome 2, is described in Poirié et al (2000). The parasitoid reference strain (Gif stock no. 486) was obtained from an isofemale strain selected from a population collected in Brazzaville (Congo), as described in Dupas et al (1998). The level of encapsulation of the 940 reference host strain parasitised by the 486 reference strain was $97.3 \%$ (675 eggs tested).

\section{Bioassay experiments}

The infestation experiments were performed at $25^{\circ} \mathrm{C}$, and the encapsulation rate was calculated as the ratio of the number of encapsulated eggs to the total number of eggs recovered (as described in Carton et al, 1992). Most of the host larvae were mono-infested. Statistical justification and details about how to calculate the encapsulation parameter can be found in Dupas and Boscaro (1999).

$D$. melanogaster and L. boulardi populations were tested after one or two generations of laboratory culture. For sympatric infestations, parasitoid populations were used to infest their sympatric host populations, and the encapsulation level was recorded (Table 1). The rate at which hosts encapsulate $L$. boulardi, which defines host resistance of local populations, was measured against the parasitoid avirulent reference strain 486 (Table 1). The rate at which $L$. boulardi parasitoids from different populations escape encapsulation, that is, parasitoid virulence, was measured against the host-resistant reference strain 940 by calculating the proportion of eggs that escaped encapsulation (Table 1).

$\mathrm{F}_{\text {st }}$ estimation procedure

Following an estimation of the frequencies of the resistance phenotype in D. melanogaster populations, allele frequencies at the resistance locus were determined according to the Hardy-Weinberg equilibrium hypothesis, assuming that a single gene (Rlb), responsible for resistance in the reference strain, is responsible for the resistance in natural populations. The $R l b$ allele encoding the resistant phenotype is dominant over the susceptible allele (Benassi et al, 1998). The $F_{\text {st }}$ values were then calculated according to Rousset (1997).

\section{Results and discussion}

Geographical patterns of resistance and virulence

Drosophila-parasitoid wasp systems are used as models for the study of immune responses in insects, but there is surprisingly little evidence that these reactions actually occur in natural populations. Most of the studies intended to investigate the geographic variation of Drosophila resistance make use of more or less avirulent strains of parasitoids that allow its expression (Kraaijeveld and Van Alphen, 1995; Kraaijeveld and Godfray, 1999). These studies, of course, do not imply that the formation of capsules around parasitoid eggs can be easily observed in the field, because virulent parasitoids can overcome the host defences. Only by means of infestation studies using sympatric host and parasitoid populations can the occurrence of parasitism failure be recorded. For example, in the interaction between D. melanogaster and Asobara tabida, the parasitoid encapsulation rate in host larvae infested by sympatric female wasps was shown to display a high level of geographic variation in Europe (Kraaijeveld and van der Wel, 1994). The first large-scale experimental survey of the encapsulation rate in sympatric host-parasitoid populations - covering the entire distribution area of the parasitoid species - is presented in Figure 2a for the L. boulardi/D. melanogaster system. Capsule formation was observed only in populations from tropical Africa, suggesting that there is a high level of parasitoid virulence and/or a low level of host resistance in the rest of the distribution area.

A clear understanding of the coevolutionary process involving resistance and virulence requires a suitable genetic model of interactions (Godfray, 2000). Generally, when only one host and one parasitoid species are considered, it is implicitly assumed that a pairwise resistance-virulence interaction does occur (Kraaijeveld and Godfray, 1999). If there is no significant local adaptation, sympatric levels of encapsulation will be predictable from the general levels of resistance and virulence in the two partners. This could be achieved by using reference strains to estimate host resistance and parasitoid virulence separately (Kraaijeveld and Van Alphen, 1994, 1995; Dupas and Boscaro, 1999).

The D. melanogaster and L. boulardi reference strains that were used were derived from natural populations, using isofemale strains and/or selection procedures. Single genes with a major effect on resistance and virulence have been identified in these strains (Dupas et al, 1998). Godfray (2000) suggested that such genes might be recessive deleterious mutants of genes that make little contribution to the additive genetic variation in natural populations. However, this seems highly unlikely. Indeed, as we will see, using these reference strains and understanding how they interact, it is possible to make accurate predictions of the encapsulation rate in sympatric host-parasitoid populations. 
Furthermore, it has been shown (Orr and Irving, 1997; Benassi et al, 1998) that increased D. melanogaster resistance to $A$. tabida in strains selected from natural populations is also attributable to a genetically simple basis and is localized on chromosome $2 \mathrm{R}$, near the centromere. The gene for resistance to A. tabida, Rat, studied from one isogenic reference strain, is located in exactly the same chromosome area (Poirié et al, 2000). To date, there is no reason to suggest that the genes responsible for host resistance and parasitoid virulence in reference strains are different from those acting in the field. Obviously, this will only be conclusively demonstrated when these genes have been cloned.

The 'gene for gene' interaction that occurs between the reference strains is shown in Figure 1. In this system, encapsulation can only occur if the parasitoid is avirulent, suggesting that geographic variations should be mainly determined by variations in parasitoid virulence. As expected, the pattern of distribution of 'sympatric' encapsulation (Figure 2a) is not related to the pattern of host resistance (Figure 2b), but it is strongly correlated to the distribution of parasitoid virulence (Figure 2c). Indeed, only virulent parasitoids are found in all tested populations except those in tropical Africa, whereas polymorphism for host resistance is widespread.

Using a Turkish strain of L. boulardi, with undefined virulence status, Kraaijeveld and Van Alphen (1995) also recorded very low levels of encapsulation in nearly all the European populations tested, most of them varying between 0 and $12 \%$. Using reference strains thus allows us to understand the encapsulation rates observed in the field for sympatric populations of L. boulardi and $D$. melanogaster, which is in agreement with the absence of local adaptation demonstrated in the A. tabida-D. melanogaster system (Green et al, 2000; Kraaijeveld and Godfray, 2001). This suggests that local adaptation is not of major importance in either parasitoid-host system, despite the different virulence mechanisms involved.

The resistance phenotype is highly polymorphic, but its distribution does not reveal any cline or understandable pattern (Figure $2 b$ ). Considering a single resistance gene, $R l b$, the $F_{\text {st }}$ value calculated was 0.137 , which is of the same order as the $F_{\mathrm{st}}$ recorded for $D$. melanogaster trinucleotide repeats (between 0.04 and 0.149; Michalakis and Veuille, 1996), and much lower than the $F_{\text {st }}$ calculated for the Adh gene (0.49; Capy et al, 1983) from a comparable geographical range (Europe, Africa and America us Europe and Africa). Several analyses have failed to demonstrate any selective process affecting the trinucleotide repeats (Michalakis and Veuille, 1996), whereas $A d h$ is thought to be subjected to strong selection (Capy et al, 1983). This suggests that the resistance gene might also be neutral in most natural populations. The occurrence of this gene at high frequencies in situations where it does not appear to confer any advantage - since only virulent parasitoids are found remains to be explained.

In contrast, the distribution of the virulent phenotype shows a clear pattern since it is almost constant in all the

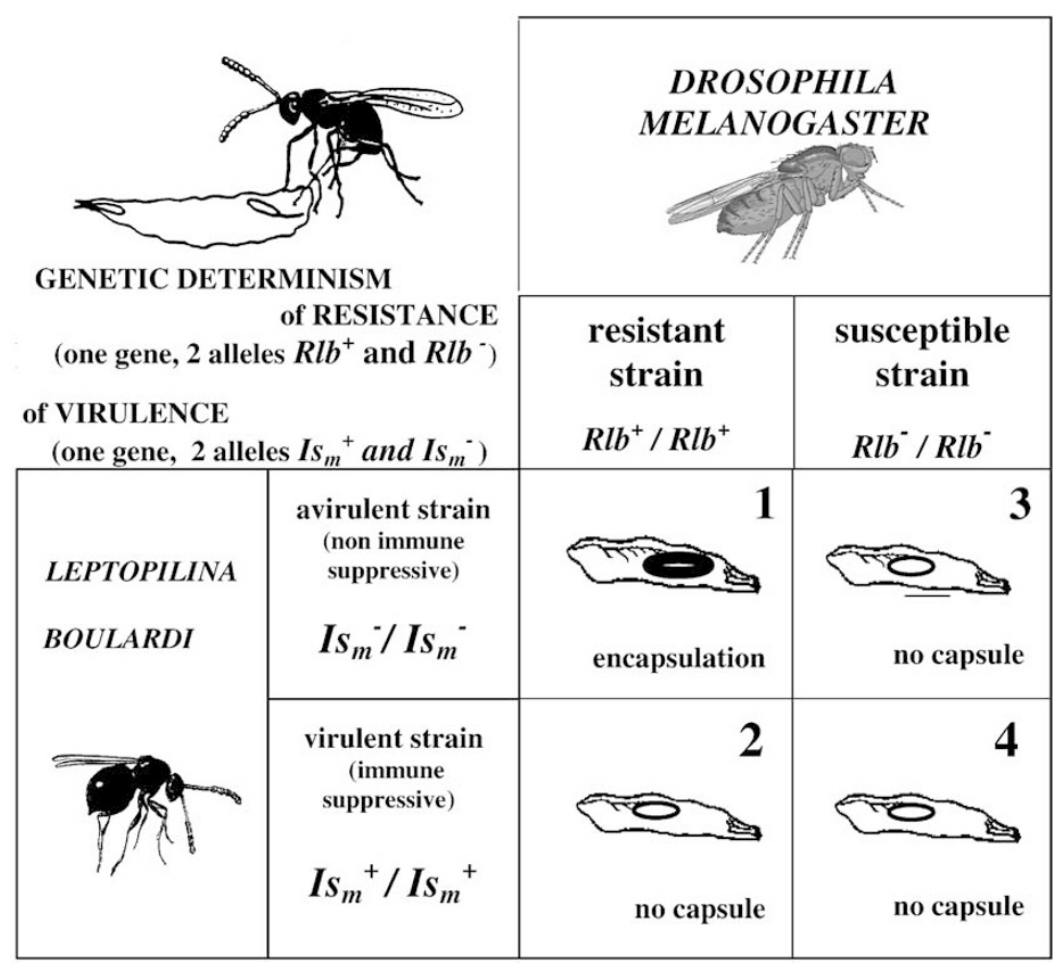

Figure 1 Matching interactions in the D. melanogaster - L. boulardi reference system. The interactions between two reference strains of the host (D. melanogaster) and two reference strains of the parasitoid (L. boulardi) are illustrated. Resistance in the host and virulence in the parasitoid are both conferred by one major gene. The host alleles $R l b^{+}$and $R l b^{-}$correspond to the resistant and susceptible phenotypes, respectively, and the alleles $\mathrm{Ism}^{+}$and $\mathrm{Ism}^{-}$are responsible for virulence and avirulence of the parasitoid. If a resistance allele and an avirulence allele occur in the host and the parasitoid, respectively (case 1), the parasitoid egg is encapsulated. In the three other situations (cases 2, 3 and 4 ), the parasitoid develops successfully. Thus, the outcome of parasitism will differ depending on whether the host or parasitoid strains differ by a single allele at the resistance or virulence loci. 
a
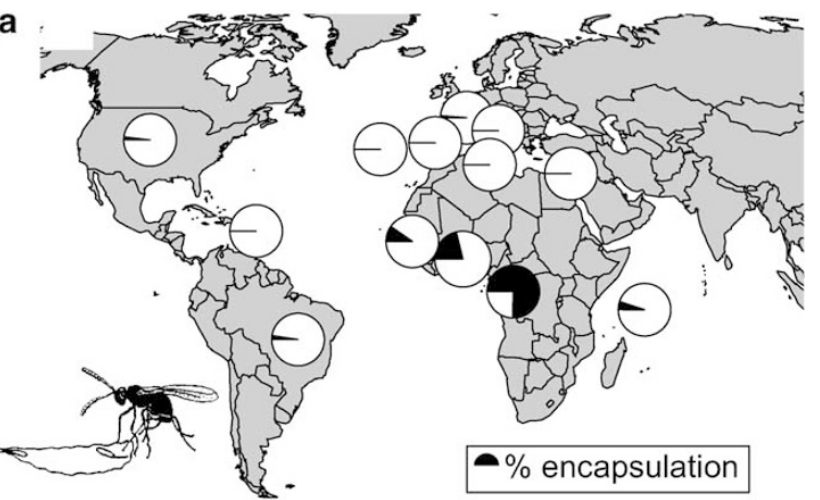

$\%$ encapsulation

b

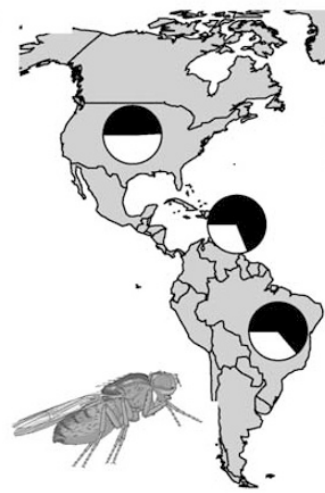

25

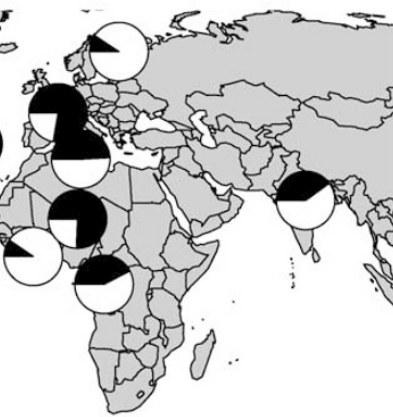

$\%$ encapsulation

C

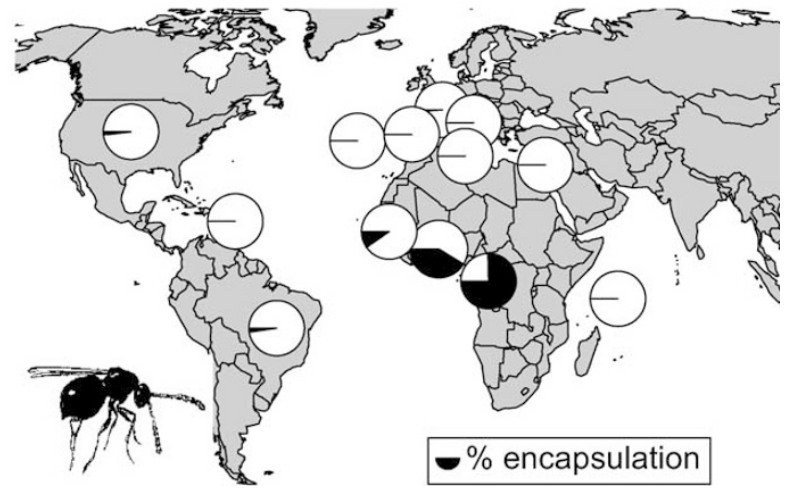

Figure 2 Geographic distribution of encapsulation rates. (a) Geographic distribution of encapsulation under sympatric conditions for D. melanogaster/L. boulardi. The rate of encapsulation (\% represented by the black portion of the pie chart) was obtained following experimental infestation of $D$. melanogaster populations by sympatric populations of L. boulardi. (b) Geographic distribution of the resistance phenotype in $D$. melanogaster populations. The resistance level (represented by the black portion of the pie chart) is estimated from the rate of encapsulation following experimental infestation of D. melanogaster populations by the reference 486 avirulent strain of L. boulardi. (c) Geographic distribution of the virulence phenotype in L. boulardi populations. The level of virulence (represented by the white portion of the pie chart) is estimated from the rate of encapsulation in experimental infestations of the reference resistant strain of $D$. melanogaster by various natural populations of L. boulardi.

populations studied, except those in tropical Africa. An evolutionary scenario, based on the specificity of the virulence gene and the structure of the host community, has been proposed to explain this pattern (Dupas and Boscaro, 1999). The factor responsible for virulence against D. melanogaster (ISm) is host specific and has been shown to be ineffective against $D$. yakuba, another African host species. Another virulence factor (ISy), nonallelic to ISm, has been found to be involved in protection against $D$. yakuba, but it is ineffective against D. melanogaster (Dupas and Carton, 1999). This relationship can be summarised as a pairwise one-gene-per-hostspecies relationship (Table 1), with a different 'virulence gene' responsible for the suppression of the immunity of each host species (Dupas and Boscaro, 1999). Although the immune suppressive genes are encoded by different loci, the parasitoid seems unable to adapt to different hosts simultaneously. The possible explanation would be the pressure of cost of immune suppression, already demonstrated for the ISy locus, that would prevent the parasitoid from acquiring many immune suppressive genes (Dupas and Boscaro, 1999). The evolution of the virulence frequency could therefore depend on the relative abundance of the target host species vs alternative hosts, mainly species of the melanogaster subgroup (Carton and Nappi, 1991). In most situations, there is at most one alternative host species to $D$. melanogaster (D. simulans), but in tropical Africa, the heart of the distribution area of the melanogaster subgroup, several alternative host species are present, including $D$. yakuba (Lachaise et al, 1988). This more diversified host community could be responsible for the lower frequency of the $\mathrm{ISm}^{+}$gene in tropical Africa (Figure 2c). The interpretation of the situation in the field was very different before the specificity of $L$. boulardi virulence factors had been demonstrated. Increased virulence against $D$. melanogaster was explained by the presence of an alternative and more resistant host species, $D$. simulans, selecting for greater overall virulence in the parasitoid (Boulétreau, 1986).

Thompson (1999) has questioned if 'frequency-dependant' selection [..] is sufficient to maintain coevolved gene-for-gene polymorphism'. Experimental data in our system confirm the predictions of theoretical models for a wide range of parameters (Leonard, 1993; Kraaijeveld and Godfray, 1999), notably the low genetic variability of parasitoid virulence compared to host resistance. It appears difficult to maintain genetic variations in virulence within populations displaying a high level of interaction with a major host species.

\section{The geographic mosaic theory}

Thompson's (1999) theory argues that coevolutionary interactions should be studied from a geographical perspective. This is based on the assumption that the characters involved in the interaction and the intensity of selection exhibit both spatial and temporal variations. The geographic structure of the interacting species would thus become a major component of the coevolutionary process (Thompson, 1994). Althoff and Thompson (1999) have recently studied populations of braconid parasitoids (genus Agathis) and of their moth hosts (genus Greya), and they have demonstrated that the parasitoid populations are more genetically structured than the host populations. They argued that this would affect the behaviour of both partners in the coevolutionary process, emphasising the importance of geographic mosaics in this process.

According to the geographic mosaic theory of coevolution, differences in the strength of reciprocal selection 
is the main factor responsible for a mosaic of hot spots and cold spots. This might apply to host-parasitoid interactions such as, for example, for virulence and resistance variations in the Asobara tabida - D. melanogaster model (Kraaijeveld and van Alphen, 1995). In northern Europe, A. tabida switches to another host, D. subobscura, that is more permissive towards this wasp species. This situation leads to lower virulence of the parasitoid against $D$. melanogaster, and to reduced resistance of D. melanogaster in northern Europe. Such a reduction in the reciprocal ecological impact would correspond to a cold spot of coevolution.

In addition, mosaics of hot and cold spots are also driven by the presence or absence of genetic variation for the trait subjected to reciprocal selection. Henter and Via (1995) and Hufbauer and Via (1999) reported that the genetic differentiation of pea aphid (Acyrthosiphon pisum) populations on different host plants affects the level of their genetic resistance towards the parasitoid wasp Aphidius ervi. Indeed, quantitative genetic studies have demonstrated significant genetic variation in the resistance and virulence of sympatric populations of hosts and parasitoids, as well as a high level of host resistance in $A$. pisum populations found on alfalfa host plants (Henter and Via,1995). In the case of $A$. pisum populations found on clover host plants, no significant genetic variation was detected, and a low level of host resistance was observed (Hufbauer and Via, 1999). The authors suggest that this low level of genetic variation for resistance weakens the host's coevolutionary potential.

\section{Host-parasitoid coevolution}

Resistance and virulence data in the D. melanogaster L. boulardi model represent different types of communities. How can they be explained in terms of the geographic mosaic theory?

D. melanogaster populations in northern Europe are not exposed to L. boulardi. Although there are other parasitoid species (such as L. heterotoma or Asobara tabida) that sometimes attack this host, they usually avoid oviposition in D. melanogaster in the presence of alternative hosts. Hence, L. boulardi, as well as these other parasitoids, are not important as selective agents on the evolution of resistance in D. melanogaster.

In regions with a Mediterranean climate (the Mediterranean region S.S. and the southern USA), L. boulardi is the dominant parasitoid attacking D. melanogaster, with populations built up during summer and parasitism reaching locally $100 \%$ in autumn. The alternative host species is $D$. simulans which is highly resistant against this parasitoid. Other larval parasitoids attack D. melanogaster (L. heterotoma, L. clavipes, L. longipes, A. tabida and A. rufescens), but they are negligible as a selective factor since more than $95 \%$ of parasitism is by L. boulardi. One would thus expect the evolution of a specific resistance in $D$. melanogaster. This on its turn will lead to an arm race, where L. boulardi will become more virulent. This situation, with populations where high reciprocal selection pressures occur, would be called a hot spot in Thompson's (1999) terminology. However, no genetic variation in virulence can be found among L. boulardi populations, indicating that at this moment, the arms race seems to have been won by the parasitoid. The question remains whether genetic variability is or will be available in $D$. melanogaster that will allow selection of resistant populations against virulent parasitoids.

In tropical Africa, D. melanogaster is always found together with one or more other members of the $D$. melanogaster sibling species group (Lachaise et al., 1988). All these species are potential hosts for L. boulardi, but $D$. yakuba and $D$. simulans are usually highly resistant against this parasitoid (Elsin and Prevost 1998). Other larval parasitoids attacking species from the D. melanogaster subgroup are near L. victoriae, Ganaspis xanthopoda, Asobara citri and other undescribed figitids. No quantitative data are available on the relative importance of these parasitoids as a source of selection for resistance in D. melanogaster. However, from a large number of collections made over a large part of tropical Africa and population studies elsewhere, we know that total parasitism in the rain forest never becomes as high as in the Mediterranean (van Alphen, personal communication). As different host species are available for L. boulardi and the host community has no dominant parasitoid species, the ecological interactions in the D. melanogaster/ L. boulardi system are relaxed. One could thus argue that tropical Africa is an evolutionary cold spot for this pairwise interaction, despite the occurrence of genetic variation both at the level of host resistance and parasitoid virulence ('genetic hot spot'). Finally, it could be suggested that this area is a hot spot for coevolution of L. boulardi and D. yakuba since D. yakuba is a dominant host species in the Congolese area, and immune suppressive ability of $L$. boulardi against this species has been observed in this region. However, more quantitative data on the relative abundance of $D$. melanogaster and $D$. yakuba in combination with the rate of parasitism by $L$. boulardi are needed.

The data presented here suggest that the existence of a pairwise coevolutionary process depends on the balance between the selective forces acting on each partner. These forces are expected to be asymmetric in the relation between hosts and parasitoids: each individual parasitoid needs a host to develop, but not all hosts are challenged by a parasitoid. If selection is much stronger in one partner than the other, then fixation of the trait can be expected, which would account for the general lack of variability in parasitoid virulence. Conversely, situations where numerous alternative host species are present will relax selective pressures on the parasitoid in each pairwise interaction, allowing the preservation of genetic variability and the existence of an evolutionary 'hot spot' at the community level. This would be the case for tropical Africa. For example, a new character called 'resistance to virulence' has been detected recently in some strains of D. yakuba, which are totally resistant to all the L. boulardi parasitoid strains tested to date (Dupas and Carton, 1999).

A recent paper by Lapchin (2002) also provides some theoretical framework to explain the geographical variation in virulence and resistance within parasitoid communities. He assumes in his model that there are two kinds of resistance in insect hosts: general resistance and specific resistance, the last one being more costly. In communities where a host is attacked by more than one parasitoid species, specific resistance will only evolve against a parasitoid species if it is a dominant mortality factor. The study of the different types of L. boulardil $D$. melanogaster communities and the analysis of the 
physiological mechanisms of resistance and virulence will provide experimental data to test these predictions.

In this model, we emphasise the equal importance of genetic aspects compared to the well-accepted ecological forces in driving evolutionary interactions. Furthermore, we demonstrate that it is very helpful to use genetically well-characterised reference strains in attempting to understand coevolutionary processes.

\section{Acknowledgements}

This work was funded by an EC grant (AIR36CT9-1433), ACC (SV7-95007023), the French CNRS-INRA programme Biodiversité-Interactions Durables and CNRS GDR 2153. We are thankful to an anonymous referee for helpful comments on the manuscript and to $\mathrm{J}$ van Alphen for useful population data regarding the occurrence of parasitoid species. Thanks to F. Frey for her skillful technical assistance.

\section{References}

Althoff DM, Thompson JN (1999). Comparative geographic structures of two parasitoid-host interactions. Evolution 53: 818-825.

Benassi V, Frey F, Carton Y (1998). A new specific gene for wasp cellular immune resistance in Drosophila. Heredity 80: 347-352.

Boulétreau M (1986). The genetic and coevolutionary interactions between parasitoids and their hosts. In: Waage JK, Greathead DJ (eds) Insect Parasitoids: Thirteen Symposium of the Royal Entomological Society of London, Academic Press: New York. pp 169-195.

Capy P, David JR, Allemand R, Hytia P, Rouault J (1983). Genetic properties of north African Drosophila melanogaster and comparison with European and Afrotropical populations. Genet Sel Evol 15: 185-200.

Carton Y, Frey F, Nappi AJ (1992). Genetic determinism of the cellular immune reaction in D. melanogaster. Heredity 69: 393399.

Carton Y, Nappi AJ (1991). The Drosophila immune reaction and the parasitoid capacity to evade it: genetic and coevolutionary aspects. Acta Oecol 12: 89-104.

Carton Y, Nappi AJ (1997). Drosophila cellular immunity against parasitoids. Parasitol Today 13: 218-226.

Carton Y, Nappi AJ (2001). Immunogenetics aspects of the cellular immune response of Drosophila against parasitoids. Immunogenetics 52: 157-164.

Dupas S, Boscaro M (1999). Geographic variation and evolution of immune suppressive genes in a Drosophila parasitoid. Ecography 22: 284-291.

Dupas S, Carton Y (1999). Two non-linked genes for specific virulence of Leptopilina boulardi against Drosophila melanogaster and D. yakuba. Evol Ecol 13: 211-220.

Dupas S, Frey F, Carton Y (1998). A single parasitoid segregating factor controls immune suppression in Drosophila. J Hered 89: 306-311.

Eslin P, Prevost G (1998). Hemocyte load and immune resistance to Asobara tabida are correlated in species of the Drosophila melanogaster subgroup. J Insect Physiol 44: 807-816.
Green DM, Kraaijeveld AR, Godfray HCJ (2000). Evolutionary interactions between Drosophila melanogaster and its parasitoid Asobara tabida. Heredity 85: 450-458.

Godfray HCJ (1993). Parasitoids Behavioral and Evolutionary Ecology. Princeton University Press: Princeton.

Godfray, HCJ (2000). Host resistance, parasitoid virulence, and population dynamics. In: Hochberg ME, Ives AR (eds) Parasitoid Population Dynamics, Princeton University Press: Princeton. pp 120-138.

Henter HJ, Via S (1995). The potential for coevolution in a hostparasitoid system. I. Genetic variation within an aphid population in susceptibility to a parasitic wasp. Evolution 49: 427-438.

Hufbauer RA, Via S (1999). Evolution of an aphid-parasitoid interaction: variation in resistance to parasitism among populations specialized on different plants. Evolution 53: 1435-1445.

Kraaijeveld AR, van Alphen JJ (1994). Geographical variation in resistance of the parasitoid $A$. tabida against encapsulation by D. melanogaster larvae: the mechanism explored. Physiol Entomol 19: 9-14.

Kraaijeveld AR, van der Wel NN (1994). Geographic variation in reproductive success of the parasitoid Asobara tabida in larvae of several Drosophila species. Ecol Entomol 19: 221-229.

Kraaijeveld AR, van Alphen JJ (1995). Geographic variation in encapsulation ability of Drosophila melanogaster larvae and evidence for parasitoid specific components. Evol Ecol 9: 10-17.

Kraaijeveld AR, van Alphen JJ, Godfray HCJ (1998). The coevolution of host resistance and parasitoid virulence. Parasitology 116: S29-S45.

Kraaijeveld AR, Godfray HCJ (1999). Geographic patterns in the evolution of resistance and virulence in Drosophila and its parasitoids. Am Nat 153: S61-S74.

Kraaijeveld AR, Godfray HCJ (2001). Is there local adaptation in Drosophila-parasitoid interactions? Evol Ecol Res 3: 107-116.

Lachaise D, Cariou ML, David JR, Lemeunier F, Tsacas L, Ashburner M (1988). Historical biogeography of the Drosophila melanogaster species subgroup. In: Hetch MK, Wallace B, MacIntyre RJ (eds) Evolutionary Biology, Plenum Press: New York. Vol 25, pp 127-173.

Lapchin L (2002). Host-parasitoid association and diffuse coevolution: when to be a generalist? Am Nat 160: 245-254.

Leonard KJ (1993). Stability of equilibria in a gene-for-gene coevolution model of host-parasite interactions. Phytopathology 84: 70-77.

Michalakis Y, Veuille M (1996). Length variation of GAG/GAA trinucleotide repeats in natural populations of Drosophila melanogaster and its relation to the recombination rate. Genetics 143: 1713-1725.

Orr HA, Irving S (1997). The genetics of adaptation: the genetic basis of resistance to wasp parasitism in Drosophila melanogaster. Evolution 5: 1877-1885.

Poiriè M, Frey F, Hita M, Huguet E, Lemeunier F, Periquet G (2000). Drosophila resistance genes to parasitoids: chromosomal location and linkage analysis. Proc $R$ Soc Lond B 267: 1417-1421.

Rousset F (1997). Genetic differentiation and estimation of gene flow from $F$-statistics under isolation by distance. Genetics 145: 1219-1228.

Strand MR, Pech LL (1995). Immunological basis for compatibility in parasitoid-host relationships. Ann Rev Entomol 40: 31-56.

Thompson JN (1994). The Coevolutionary Process. University of Chicago Press: Chicago.

Thompson JN (1999). Specific hypotheses on the geographic mosaic theory of coevolution. Am Nat 153: S1-S14. 